\title{
LAS INVESTIGACIONES FITOQUIMICAS Y SUS RELACIONES CON LOS HERBARIOS
}

\author{
Jorge S. Marroquín* \\ Xorge A. Domínguez**
}

\subsection{ANTECEDENTES}

Con motivo del IV Centenario de la Universidad de México (1551-1951) hoy Nacional Autónoma, se llevó a efecto el Congreso Científico Mexicano, cuyas Memorias distribuidas en 15 tomos abarcan todas las áreas mayores del saber humano. Para el año de su publicación, 1953, bueno es hacer notar que la Botánica ocupa un lugar importante, a juzgar por los trabajos publicados en el tomo VI. Ahí mismo, Débora Ramírez Cantú (109) explica el porqué de la existencia del herbario de la Universidad, cuál es su función y qué desarrollo deberá alcanzar en el futuro. A 20 escasos años de esas apreciaciones, nos reunimos en este Coloquio para revisar los logros, conocer nuestras potencialidades en las investigaciones botánicas y tratar de entrever el futuro en función de las necesidades del país.

Pues bien, en esta ponencia para la que hemos sido invitados, daremos un vistazo a los aspectos fitoquímicos, conscientes de que esta es una de las áreas de la investigación que se ha desarrollado con mayor rapidez en México. Hemos suprimido el añadido del campo de la farmacología, que originalmente se incluía en el título asignado, por tratarse de otro vasto campo científico. A este respecto, en los tomos dedicados a las Ciencias Médicas del aludido Congreso Científico Mexicano, solamente dos contribuciones aparecen en el área de la farmacología $(70,92)$.

En el VI Simposio Internacional sobre la Química de los productos naturales (esteroides y terpenos), los investigadores mexicanos en el área de la Fitoquímica tuvieron una activa y destacada participación; tal evento se desarrolló del 21 al 25 de abril de 1969 y cinco meses después tenía lugar el IV Congreso Mexicano de Botánica en el norte del país.

* Colegio de Graduados, Escucla Superior de Agricultura “Antonio Narro", Universidad Autónoma de Coahuila. Buenavista, Saltillo, Coah.

** Departamento de Química, Instituto Tecnológico y de Estudios Superiores. Monterrey, N. L. 
En los cinco congresos mexicanos de Botánica, incluido entre ellos el primero latinoamericano del año pasado, prácticamente no se han presentado trabajos de naturaleza fitoquímica, si bien es cierto que algunos han versado sobre aspectos fisiológicos y etnobotánicos. ${ }^{1}$

Entre las actividades conjuntas que llevan al cabo los botánicos y los químicos, cabe mencionar la que Bruhn (13) llama "el complejo de los peyotes", o sea la acción combinada de los cactólogos, los fitoquímicos, los etnólogos y los psicólogos, entre otros, quienes han desatado una verdadera "cacería de alcaloides" $(90,154,155)$ en especial sobre las Cactáceas $(1,2,3,46))$. Se buscan los principios psicoactivos y nuevas substancias químicas (12). La investigación básica proporciona así los cimientos de una edificación técnica en materia de aprovechamiento de nuestros recursos vegetales. Sin embargo, desde fines del siglo pasado, como es sabido, el Instituto Médico Nacional se ocupaba ya de las investigaciones fitoquímicas. ¿Quién no habrá oído mencionar o habrá visto citas de los pioneros en este campo de la ciencia como Fernando Altamirano, José Ramírez, José Terrés, Manuel Toussaint, Eduardo Armendáriz, Domingo Orvañanos y Secundino Sosa? ¿E igualmente de Francisco Río de la Loza, Donaciano Cano, Alfonso L. Herrera, Mariano Lozano, Federico Villaseñor, Miguel Zúñiga, Daniel Vergara, Juan Govantes, Joaquín Huici y Roberto M. Jofre?

Estos precursores de la fitoquímica en México, aparecen mencionados en la obra publicada por la Secretaría de Fomento en 1894 y 1895, como "Datos para la preparación de la Materia Médica Nacional" (7). Nos hablan de las plantas conocidas como el "inguande", la "contrayerba blanca", "el pipitzahoac", "el pañete", "el yoloxóchitl", "la zábila", "la yerba de la puebla", "el zoapatle", "el chicalote", "el tlacoxiloxóchitl", "el cuauchichic", "el matarique", "la raíz del oso", "la guapilla", "la pingüica", "el tlalocopetate", "la sangre de toro", "la yerba del tabardillo", "La Atanasia amarga", "el tumbavaqueros", "la sangre de drago", "el simonillo", "el añil", "el yoyote", "la yerba del pollo", "los cuajiotes verde y chino", "el árbol del Perú", "el chilpanxóchitl" y "el cozticpatli".

Recientemente muchos trabajos se han realizado sobre algunas de estas plantas gracias a las técnicas modernas y al instrumental necesario en nuestras instituciones, como veremos adelante.

1 Ver los resúmenes de los trabajos presentados en los Congresos de Botánica, prepa. rados por la Sociedad Botánica de México, a saber: 17-21 de Sept, 1963 en San Luis Potosí; 24-28 de Oct. 1966 en México, D. F.; 8-11 de Sept. 1969 en Monterrey, N. L. y Saltillo, Coahuila; 3-9 de Dic. 1972 en México, D. F. 


\section{FITOQUIMICA Y HERBARIOS}

\subsection{LOS HERBARIOS}

Ha quedado de manifiesto en el desarrollo de este Coloquio que la función básica de un herbario es proporcionar material comparativo para la identificación de las plantas.

El valor de las colecciones herborizadas aumenta cuando éstas han sido y son escrutadas por los botánicos especialistas. El herbario representa así un cúmulo de información que sirve de referencia permanente a los científicos en la preparación de revisiones, monografías taxonómicas, sinopsis y trabajos florísticos regionales $(8,9,109,110)$.

Pero además otros científicos y técnicos, así como los profesores y los estudiantes avanzados, encuentran a menudo en el material de herbario una base de sustentación, un punto de partida para llevar adelante con seguridad sus pesquisas de carácter aplicado. Se familiarizan con los taxa involucrados mediante la adquisición de los datos generales, ecológicos y de distribución geográfica que cada etiqueta usualmente posee y en fin, los conduce a las referencias especializadas para "rastrear" cada taxón en la literatura.

El herbario asume así su pleno valor de acervo científico; cumple con la función de servir un mayor número de propósitos, entre ellos el de proporcionar material fidedióno para los muestreos de los pólenes, como depósito permanente de los tipos taxonómicos, e inclusive como una fuente de datos históricos $(9,62,74,150)$.

Desde el punto de vista de la fitoquímica, ¿qué importancia reviste un herbario? La respuesta es obvia: reunir y proteger todos aquellos ejemplares que sean representantes adecuados para la identificación del voluminoso material botánico que se está analizando químicamente. Tales ejemplares servirán de referencia y quedan ubicados como comprobantes ("vouchers") de los estudios fitoquímicos de ellos derivados. Sin embargo el simple "depósito" de testigos no garantiza el cuidado profesional que se exige; este aspecto debe vigilarse celosamente. Es evidente que la investigación fitoquímica debe de tener como base un correcto planteamiento taxonómico: ¿de qué taxón se trata? ¿ha sido bien determinado? ¿corresponde a la región geográfica explorada - como especie nativa - o es un reciente invasor? Estos detalles ameritan la puesta en práctica de todos los recursos de la buena investigación. La concurrencia coordinada de los químicos y los botánicos sirve al propósito $(10,66)$.

La existencia y buen nombre de un herbario, en este caso fanerogámico, pero extensivo a los criptogámicos también, implica, para su arreglo, su ubicación, su manejo, su operación (servicio) y su crecimiento, el cumplimiento de cier- 
to cánones que van desde la competencia profesional del personal que lo dirige hasta el adiestramiento de quienes ejecutan la obra manual rutinaria del montaje, etiquetado y distribución de los ejemplares en los anaqueles.

Los botánicos profesionales que atienden y trabajan sobre cierto grupo de plantas por lo general elevan la calidad del herbario, ya que contribuyen a la actualización nomenclatural de las colecciones y aseguran la determinación correcta de los especímenes recibidos para consulta o bajo el sistema de intercambio $(9,62,195)$.

Los herbarios deben contar con colectores eficientes que combinen las tareas de surtir de ejemplares vivos a los jardines botánicos con la de preparación de muestras prensadas para herbario. Esta doble actividad es deseable para las instituciones que imparten carreras de índole agronómica (58), pero requieren partidas presupuestales permanentes relativamente altas.

Es muy alentador saber que las instituciones mexicanas están siendo consideradas como confiables y serias para recibir los "préstamos" de ejemplares a revisión, e incluso de "tipos", cuyo manejo reviste gran cuidado y responsabilidad. Los botánicos especialistas van creando esta atmósfera de respetabilidad profesional que reditúa a la postre una magnífica herencia científica y relaciones de alto nivel. Esto se refleja en la calidad de los trabajos que se presentan en los Congresos y en las publicaciones del ramo.

Es difícil todavía en la actualidad encontrar personal interesado en la realización de trabajos sobre grupos botánicos poco estudiados, y aunque a veces se inician en esta área, pocos logran estabilizar sus inquietudes taxonómicas como para obtener frutos a corto plazo; esto es explicable en virtud del escaso número de instituciones que patrocinan programas de carácter botánico puro. A pesar de ello, el desenvolvimiento de la botánica en México es notable, a raíz del resurgimiento de las ciencias naturales en el panorama científico nacional, y con una señalada influencia del vigor demostrado en otros países hacia las ciencias biológicas en particular. Esto motiva, en parte, a las nuevas generaciones a no orientrse tan sólo hacia las áreas más tradicionales de realización profesional, sino que también estimula la búsqueda de campos menos competitivos, o sean aquellas carreras avaladas por las urgencias propias del desarrollo de nuestro país $(141,142)$.

\subsection{LA FITOQUIMICA}

En el último cuarto de siglo ha surgido en México un vigoroso grupo de investigadores sobre aspectos fitoquímicos; muchos de ellos de reconocido pres. 
tigio tanto nacional como internacional. Han desarrollado una fructífera labor científica al grado de abrir nuevas perspectivas a la industria y a la medicina $(65,81,141)$.

Las familias botánicas que mayores inquietudes y atención han despertado en ellos son, a saber:

$\begin{array}{ll}\text { Amarilidáceas (incluyendo Agaváceas) } & \text { Gencianáceas } \\ \text { Amarantáceas } & \text { Labiadas } \\ \text { Apocináceas } & \text { Leguminosas } \\ \text { Aristoloquiáceas } & \text { Liliáceas } \\ \text { Asclepiadáceas } & \text { Litráceas } \\ \text { Cactáceas } & \text { Lorantáceas } \\ \text { Cariofiláceas } & \text { Malpigiáceas } \\ \text { Celastráceas } & \text { Papaveráceas } \\ \text { Compuestas } & \text { Ramnáceas } \\ \text { Convolvuláceas } & \text { Rutáceas } \\ \text { Cucurbitáceas } & \text { Solanáceas } \\ \text { Dioscoreáceas } & \text { Teofrastáceas } \\ \text { Euforbiáceas } & \text { Verbenáceas } \\ \text { Garriáceas } & \end{array}$

Las siguientes familias han merecido atención en otras épocas:

$\begin{array}{ll}\text { Anacardiáceas } & \text { Loganiáceas } \\ \text { Bromeliáceas } & \text { Magnoliáceas } \\ \text { Comelináceas } & \text { Plumbagináceas } \\ \text { Coriariáceas } & \text { Polemoniáceas } \\ \text { Ericáceas } & \text { Ranunculáceas } \\ \text { Esterculiáceas } & \text { Valerianáceas } \\ \text { Leguminosas } & \text { Zigofiláceas }\end{array}$

Lobeliáceas

En otros países los químicos han hecho (y prosiguen) estudios sobre éstas y/u otras familias, por ejemplo las Berberidáceas para alcaloides (18, 20, $23,68,104,151)$ y antocianinas; las Ranunculáceas en serología con propósitos sistemáticos (75); las Compuestas por algunas de sus propiedades insecticidas (35); Mirtáceas (fundamentalmente Eucalyptus), Pináceas y otras coníferas 
para terpenos; Gutíferas, Canelláceas, Poligonáceas, Rubiáceas, Simaroubáceas, etc., por sus propiedades insecticidas (103); las Crucíferas para glucosinolatos y aceites mostaza (4).

La Asociación Americana de taxónomos de las plantas distribuyó recientemente entre sus asociados un manual técnico donde se explican los procedimientos a seguir - y el equipo indispensable - necesarios para conducir estudios fitoquímicos (149). Que los propios botánicos se hayan preocupado por ello es lo que más llama la atención; es evidente que este campo fronterizo e interdisciplinario está en auge a nivel internacional. Su despertar en México nos da idea de que los investigadores mexicanos van acordes con este sentir, dado el volumen de trabajos realizados y publicados en estos últimos años.

La secuencia sugerida en los estudios de índole fitoquímica ha sido dada por varios autores, entre ellos Rosenthaler (139), Domínguez (37, 55), Dakshini et al. (24), Raffauf (107) y otros.

Varios países cuentan con institutos destinados al estudio de los recursos naturales vegetales, sobre todo de aquellas plantas que aportan principios susceptibles de estudio en las ciencias médicas; algunos productos naturales han sido la clave y la razón de la expansión de la industria farmacéutica (81). Guerra (70) señala que para emprender el estudio de diversos grupos de drogas mexicanas, iniciado en 1939, llevaba en mente la posibilidad de encontrar productos de aplicación terapéutica.

Esto lo llevó a levantar un inventario bibliográfico de la Materia Médica Mexicana en 1950 (69), que constituye la base del trabajo experimental. En sus investigaciones divide las drogas indígenas en los grupos siguientes: antibióticos, parasiticidas, digestivos, cardiotónicos, bociógenos, antidiabéticos y fantásticos.

Varias especies las consideró para la extracción de principios bacteriostáticos, entre ellas Parthenium hysterophorus, Loeselia coccinea, Eysenhardtia amorphoides, Piqueria trinervia, Buddleia americana y otras (70). Un trabajo similar sobre la determinación de la acción antibiótica de 92 plantas mexicanas fue realizado por Domínguez et al. (38). Domínguez y Sierra (49) aislan un nuevo alcohol diterpénico y partenina de la "cicutilla" o "yerba amargosa" Parthenium hysterophorus; dicha partenina, un guaianólido, fue caracterizada por Hertz y Watanabe en 1959 (71).

Posteriormente, la partenina ha sido probada como agente inhibidor del crecimiento en plántulas de frijol (117).

En la década de los años " 50 ", el Instituto Nacional de Cardiología inició estudios sobre plantas de posible acción cardíaca. Méndez (92) considera que 
los proyectos de este carácter requieren, además de la colección del material botánico y su identificación, la extracción química de sus principios activos, los estudios farmacológicos y taxicológicos y como meta final su estudio clínico. Plantas de los géneros Talauma, Thevetia y Plumeria fueron objeto de estudio, las que ya eran consideradas por la tradición popular como plantas de posible acción cardíaca. Rivera (111) describe los usos y las propiedades medicinales de varias especies del área de Izúcar de Matamoros, Pue.

Los principios tóxicos de las plantas, especialmente los de las llamadas plantas venenosas, son de muy diversa naturaleza química.

Se les agrupa en cuatro grandes categorías:

a) Inhibidores enzimáticos,

b) Irritantes fisiológicos,

c) Alérgenos,

d) Modificadores de sistemas hormonales.

Entre los inhibidores enzimáticos se incluyen las proteasas, las colinesterasas y los agentes cianogenéticos, pero sin duda los más famosos son los alcaloides, a pesar de que no son los responsables, en la mayor parte de los casos, de la naturaleza tóxica de las plantas.

Los estudios sobre alcaloides se incrementaron en nuestro país desde que el Instituto de Química de la U. N. A. M. se localizaba en Tacuba, D. F. (141). Abundaron los trabajos en colahoración entre los químicos mexicanos y algunos extranjeros, muchos residentes en México. Así por ejemplo, habiendo repercutido notablemente el éxito de los estudios sobre la fitoquímica de Rauwolfia serpentina de la India, en México se condujo un estudio sobre Rauwolfia heterophylla, especie mesoamericana, de material oaxaqueño; de ambas especies se ha aislado reserpina $(27,31)$. Djerassi et al. (31) encontraron tres alcaloides de $R$. heterophylla.

Los estudios sobre alcaloides también se efectuaron en varios géneros de Cactáceas, especialmente de la subtribu Cereanae. Existen estudios realizados por Roca (113) y por De Lille $(25,26)$ sobre los "órganos", tan propios del paisaje rural del altiplano; posteriormente Djerassi y colaboradores buscaron principios en diversas suculentas, así aislaron pilocereina del "órgano" Pachycereus marginatus, alcaloide ya conocido de Lophocereus schottii $(28,32)$. Las especies de Lemaireocereus mostraron riquezas en saponinas triterpenoides, mas no revelaron alcaloides $(32,33)$. Un alcaloide denominado cuauchichicina fue aislado de Garrya laurifolia (29). Djerassi et al. (30) aislaron de Iresine celosioides la iresina, una sesquiterpenlactona, y confirmaron la ausencia de al- 
caloides de esta planta. Por la misma época, Djerassi et al. (32) reunieron material de cactáceas de Chile, Perú, Venezuela y México y procedieron a estudiar la fitoquímica de los géneros Trichocereus, Escontria y Lemaireocereus, así como la Malpigiácea Byrsonima (el "nance" o "nanche" común en las llanuras de sabanas tropicales del sureste de México) y Cucurbitáceas del género Luffa.

Con respecto a los "garambullos", Djerassi y su equipo (33) no encontraron alcaloides pero aislaron siete triterpenos, de los cuales sólo dos se han encontra. do fuera de la familia de las Cactáceas. Los autores consideraron que el propósito principal de sus estudios era el aislamiento y prueba estructural de nuevos alcaloides y triterpenos "hasta donde fuere posible", dado que sus resultados entreven un doble interés: taxonómico y biogenético.

Se sabe que todos los "garambullos" (Myrtillocactus) son ricos en glicósidos; en cambio no revelaron alcaloides, confirmando así la regla conocida de que "los glicósidos y los alcaloides por lo general no ocurren juntos en la misma planta".

Además de sus resultados sugirieron una diferenciación taxonómica con base química entre Myrtillocactus grandiareolatus y M. geometrizans.

Agurell $(1,2)$ y Agurell et al. (3) han contribuido al conocimiento de los alcaloides de varios géneros de Cactáceas, destacando el género Trichocereus.

Domínguez et al. $(44,46,50)$ han estudiado Cactáceas del Noreste de México, particularmente las globulares, de las que aislaron un nuevo hidroxicolesterol llamado "itesmol"; un triterpenodiol, el eisacol; un nuevo flavonoide, la retusina y localizaron alcaloides del tipo de tetrahidro-isoquinolina en 16 Cactáceas. Al respecto, otros autores se han ocupado también de los alcaloides de estas suculentas, así sea a modo de revisión bibliográfica o de búsqueda química, entre quiene destacan Bruhn $(12,13,14)$ y Sodi-Pallares (147, 148).

El interés por los alcaloides es universal, y sobre ellos se han escrito muchos trabajos, por ejemplo los de Schultes (143, 144), Raffauf (108), Cava (16, 17, 18), Cruse (21). Walls et al. (153), Li y Willaman (83). Willaman y Schubert (154. 155). Pero más bibliografía y riqueza de datos sobre plantas mexicanas se encuentran en el libro de Langman (82). Dignas de mención se consideran las especies del género Stemmadenia (Apocynaceae) del trópico mexicano, como fuentes de alcaloides, aislados por Walls, Collera y Sandoval (153).

De gran significado taxonómico y sistemático han resultado las betacianinas, de distribución más bien localizada entre las angiospermas (85).

En cuanto a otros principios químicos de relevancia, en las Compuestas Flores y Herrán (60) establecieron la estructura de una nueva flavona a partir de Brickellia pendula, a la que atribuyen propiedades medicinales y cuyos an. 
tecedentes químicos se remontan hasta 1894 cuando Río de la Loza estudió Atanasia amarga (Brickellia squarrosa). De esta planta se aisló por Flores y Herrán (61) la atanasina, un flavonoide.

Para los años de la década de los " 60 ", se intensificaron los estudios fitoquímicos sobre las Compuestas, principalmente entre los investigadores del Instituto de Química de la U.N.A.M. ahora ampliados a otras instituciones, como el I.P.N. y el I.T.E.S.M. ${ }^{2}$

Herz et al. $(72,73)$ inician en Florida una serie de estudios sobre el género Helenium, seguidos por Romo, Joseph-Nathan y Díaz (124, 125), quienes proponen la aromatina y la aromaticina, dos nuevas lactonas sesquiterpénicas, cuyas estructuras establecen.

Los trabajos se multiplican en el Instituto de Química de la Universidad Nacional que pronto se ubica en la Torre de Ciencias de la Ciudad Universitaria y consolida un equipo de investigadores. Destacan las pesquisas sobre Cacalia $(78,115,126)$ que iniciara hace muchas décadas el insigne Altamirano (7). Del género Ambrosia Romo et al. (127) aislan muchos principios y reconocen sus estructuras, por ejemplo de la cumanina; nuevos pseudoguaianólidos aislados de Franseria y Ambrosia (129), los guaianólidos de Ambrosia cumanensis (131), la distribución de sesquiterpen-lactonas (132), la canambrina, una sesquiterpen-lactona (134) y muy recientemente Payne $(99,100)$ et al. revelan la quimosistemática y taxonomía de Ambrosia Chamissonis y estudian los aceites volátiles en el género Ambrosia. Caballero y Walls (15) estudian los productos naturales del "zoapatle" Montanoa tomentosa, otrora estudiada por José Ramírez. E. Domínguez y J. Romo (34) caracterizan nuevas sesquiterpen-lactonas relacionadas con la tenulina de Helenium mexicanum. Otro género importantísimo en las Compuestas es Eupatorium. Romo et al. (130) estudiaron E. ligustrinum, del que aislaron un guaianólido, la ligustrina. Otros géneros que merecieron su atención son Zaluzania (128), Artemisia y Chrysanthemum (133, 137), la xerofita Parthenium argentatum ("guayule") del que aislaron las guayulinas "A" y "B", nuevos sesquitterpenos (135) así como otros géneros (138).

Por su parte Estrada et al. (59) y Rodríguez Hahn (116) hacen estudios fitoquímicos sobre Flourensia resinosa, aislándose en este mismo año un nuevo triterpeno, la resinona. En tanto que Romo de Vivar ha realizado estudios en colaboración con otros investigadores del mismo Instituto de Química, sobre Helenium (118), Achillea (120), Parthenium confertum (121) y Eupatorium glabratum (122).

\footnotetext{
2 T.N.A.M.: Universidad Nacional Autónoma de México.

T.P.N.: Instituto Politéruico Nacional (Centro de Investigación y de Estudios Avanzados).

I.T.E.S.M.: Institutn Tecnológico y de Estudios Superiores de Monterrey.
} 
El año pasado, Romo de Vivar et al. (1.23) estudiaron los triterpenoides de la Celastrácea del norte de México Mortonia greggii ("afinador"). Ríos et al. (112) aislan la stevina, un pseudoguaianólido de Stevia rhombifolia. Otra especie de este género, Stevia berlandieri, se está estudiando en Monterrey.

Joseph-Nathan se interesa por el género Perezia (80) y por Cacalia (78), y analiza algunos sesquiterpenos por RMN doble, contribuyendo al conocimiento de la química de la perezona $(76,77,79)$.

Ortega et al. (95) separan la odoratina, un pseudoguaianólido de Hymenoxys odorata, planta venenosa del norte de México. Posteriormente Ortega et al. (96) aislan orizabina y zexbrevina " $\mathrm{B}$ ", nuevos germacranólidos; y en este año, Ortega et al. (97) estudian los componentes de Zexmenia brevifolia, caracterizando la estructura de zexbrevina "C".

De donde se advierte un aumento considerable de los artículos publicados anualmente por el Instituto de Química de la U.N.A.M., Institución que rebasa ya la cifra de 350 contribuciones hasta hoy publicadas en las diversas revistas periódicas del área de la fitoquímica, como Tetrahedron, Tetrahedron Letters, el Boletín del Instituto, hoy suspendido, la Revista Latinoamericana de Química, Phytochemistry: Ciencia (México), Revista de la Sociedad Mexicana de Química, Planta Medica, Economic Botany, Lloydia y otras.

Domínguez et al. (39) estudian los alcaloides de Bocconia latisepala; del "cenizo" Leucophyllum texanum ${ }^{3}$ aislaron un nuevo lignano (45) el regiomontano. De Pluchea odorata separaron dos nuevos sesquiterpenoides la cuauhtemona y un diéster de ésta. De Eupatorium havanense separaron el pulquerrol, un triterpeno aislado por primera vez por este grupo de investigadores de la "flor de nochebuena" Euphorbia pulcherrima. De Gaillardia mexicana obtuvieron una nueva sesquiterpenlactona, la neolonina con potente actividad citotóxica.

Respecto a las especies con estas propiedades, Domínguez (4.8) realizó una comparación de las substancias citotóxicas de origen vegetal a partir de plantas mexicanas usadas contra el cáncer.

Domínguez et al. (51) etudiaron asimismo las propiedades físicas de los aceites esenciales de cinco Compuestas. Manjarrez y Medina (88) etudiaron los aceites esenciales volátiles de las hojas de Artemisia mexicana y $A$. klotzschiana, los "estafiates" del norte del país. La mayoría de los aceites esenciales incluyendo aquellos de Artemisia spp. tienen además de monoterpenos, compuestos tales como lactonas, cumarinas, sesquiterpenos y otras. Son fuentes naturales de alcanfores. Manjarrez y Mendoza (89) separaron aceites esenciales de las Labiadas Cunila lythrifolia y Agastache mexicana.

\footnotetext{
3 Leucophyllum frutescens (Ter et Berl.) I. M. Johnston.
} 


\subsection{PERSPECTIVAS}

A nivel mundial el estudio de los aceites esenciales tiene buen futuro.

El Dr. Moss, de Inglaterra, se ha expresado al respecto en el sentido de que se necesitan cada vez más compuestos específicos.

Admite que las plantas tienen "ventaja" sobre el químico en la generación de compuestos "en traza" que dan una particular fragancia o aroma y añade (94) "los aceites esenciales son una fuente importante de moléculas complejas". Es preciso el uso de las fuentes naturales si es que van a usarse tales aceites esenciales como materia prima, dado que no siempre es posible sintetizarlos. Los procesos por los cuales las plantas generan estas moléculas complejas pueden a menudo relacionarse con nuestros conocimientos de taxonomía vegetal (un aspecto resulta complementario del otro). Esto sugiere a Moss (94) que las familias botánicas y las especies que pudieran tener un valor potencial se investiguen si se está buscando algún compuesto en particular $(21,65,75,81,86,90$, $98,152)$. Otra área que se ha abierto a la investigación en fitoquímica y en toxicología de insectos es la relacionada con la aplicación de mono y sesquiterpenoides al control y combate de plagas. Asimismo, el área de las hormonas de insectos y compuestos relacionados va tomando ciertamente un lugar destacado en las investigaciones a futuro. Otro grupo de compuestos de importancia extraordinaria es el de los sesquiterpenoides citotóxicos; éstos pueden tener aplicación en la quimioterapia del cáncer, de modo que deben buscarse terpenoides específicos $(38,122,48,94,149)$.

El ácido abscísico es una hormona vegetal de importancia; el Dr. Moss (94) concluye que bien pudiera haber análogos de este ácido aún más activos biológicamente y que están por descubrirse. La fitoquímica contribuye así al desarrollo de ramas conexas como la fisiología vegetal, la fisiología celular, la sistemática y la quimotaxonomía $(4,5,6,57,64,84,85,93,147)$. La aparición de la revista periódica Biochemical Systematics a nivel internacional es un buen índice del estado actual de esta área de la ciencia. Y en cuanto a la farmacología y aprovechamiento de los recursos bióticos con fines industriales, las plantas seguirán siendo la materia prima para varios tipos de industria $(14,15,16,21$, $36,42,47,48,55,65,70,81,82,83,91,94,103,143,144,145,146,154,155)$.

Aparte de las alusiones hechas anteriormente acerca de la quimotaxonomía, es del caso enfatizar que varios químicos le conceden un mayor valor predictivo a los arreglos sistemáticos basados en la distribución de muchos principios químicos en las pantas, que a aquellos sistemas construidos con bases esencialmente biológicas $(64,84,93)$. 
La XI Reunión Internacional de la Sociedad Fitoquímica de Norte América, celebrada en Monterrey, N. L., del 6 al 9 de octubre de 1971, trajo renombradas personalidades a nuestro país. Los químicos nacionales y los visitantes presentaron 42 trabajos en total, de los cuales varios trataron sobre las posibles relaciones filogenéticas entre taxa con principios químicos comunes, por ejemplo "los terpenos y la filogenia"; "la sistemática bioquímica en leguminosas del género Thermopsis" (enfatizando la química de flavonoides), "las variaciones no genéticas de los terpenos en Hedeoma", "relaciones biosintéticas en lactonas sesquiterpénicas", "la química de sesquiterpenos de plantas mexicanas" y muchos otros artículos de gran interés fitoquímico. ${ }^{4}$

Un año después, botánicos de la Universidad de N. León enviaban una tonelada de material seco de la "granadilla" Heimia salicifolia (Lythraceae) a Filadelfia para la continuación de estudios anteriores sobre los alcaloides de esta planta, de tan amplia distribución geográfica. Los estudios preliminares se habían efectuado en 1964. (11) a partir de material colectado en los alrededores de San Luis Potosí, S. L. P. De Heimia se han aislado cinco alcaloides, a saber: lythrina, cryogenina sinicuichina, heimina y sinina. Sin embargo, Douglas et al. (57) discuten la presencia de otro alcaloide, la vertina, común a H. salicifolia y a $H$. myrtifolia, lo que va de acuerdo con la consideración de que Heimia es un género monotípico.

Este prospecto bibliográfico, que de ningún modo pretende ser exhaustivo, ha tenido por objeto únicamente dar un vistazo panorámico acerca de la fitoquímica y los herbarios. Además nos lleva a suponer la existencia de una gran potencialidad de las reservas naturales del país, interpretadas a través de la fitoquímica. Esta búsqueda continua de substancias de posible significación farmacodinámica alcanza el éxito a menudo, en parte gracias a la intuición basada en la experiencia de los químicos.

Después de que el químico descubre sustancias cuya identidad establece, el farmacólogo estará en posibilidades de probarlas mediante los bioensayos, con interpretación estadística de por medio, antes de poder asegurar su significado médico y comercial. Pero esta secuencia un tanto simplista conlleva una interpretación múltiple, bioquímica y fisiológica, toxicológica y clínica, de cuyo éxito y tino depende el valor que esas sustancias adquieran para el bien de la humanidad. Aquí la ciencia alcanza sus más altos niveles de responsabilidad tanto técnica como humanitaria. Por ello, así como se habla del aumento de los precios "en cascada", así también existe la investigación científica en cascada, conca-

4 Ver resúmenes mimeografiados de los 42 trabajos en el folleto al efecto editado por el Depto. de Química del I.T.E.S.M. Sucursal de Correos "J", Monterrey, N. L. 
tenación de eventos a todas luces deseable. ¿Hay alguno de esos aspectos que tenga la prioridad? ¿Podemos suponer un principio obligado y un fin? La trabazón entre las ciencias no nos permite contestarlas afirmativamente.

Antes de concluir señalaremos que la relativa falta de ejemplares de herbario en ciertos grupos de plantas, por ejemplo las cactáceas, nos hace pensar en la conveniencia de abrir proyectos de mejoramiento y enriquecimiento de los herbarios, v. gr. mediante la colección de "topotipos"; los ejemplares así logrados podrán distribuirse a ciertos herbarios del país que garanticen su cuidado y sabio aprovechamiento. Igualmente de interés es la adquisición de información acerca de las colecciones de plantas mexicanas colectadas en épocas pretéritas y que fueron depositadas en herbarios del extranjero. De ahí el interés por cierto tipo de publicaciones, aparentemente de exclusivo valor histórico, pero que en un momento dado nos permiten llegar a tipificar taxa de dudosa filiación o confusa nomenclatura $(9,66,74,150)$.)

\subsection{RESUMEN}

Hemos visto que en los últimos 25 años se han incrementado notablemente en México los estudios químicos de las plantas, a la par que en muchos países altamente desarrollados. Ese surgimiento de los estudios fitoquímicos estimula los taxonómicos, biosistemáticos, florísticos, ecológicos y fitogeográficos, en virtud de que siempre puede presentarse la necesidad de efectuar colecciones masivas, regionales o nacionales para cuyo éxito se requiere todo tipo de información. Así los trabajos en fitoquímica generan indirectamente mayor interés por la buena práctica taxonómica, favorecen las investigaciones en equipo, pueden movilizar programas agronómicos de cierta urgencia (cultivos intensivos por ejemplo), sirven la mesa al farmacólogo, y dejan abiertos vastos campos de la investigación pura y de la aplicada a la medicina, la veterinaria, la toxicología y la industria. Los herbarios juegan el importante papel de bancos de información.

\section{AGRADECIMIENTOS}

El autor principal (J. S. M.) expresa las gracias cumplidas a los organizadores de estos coloquios sobre "Los Herbarios y su papel en el desenvolvimiento tecnológico y científico de México" por haberle extendido la invitación a participar como ponente. Al mismo tiempo agradece en forma especial las 
atenciones recibidas por parte del Dr. Jesús Romo y personal de la biblioteca del Instituto de Química de la Universidad Nacional Autónoma de México por facilitar la literatura necesaria en la preparación de este trabajo. Extiende además su agradecimiento al comentarista de esta ponencia Dr. Pedro Joseph-Nathan del Centro de Investigación y de Estudios Avanzados del I.P.N. por haber proporcionado valiosa información. Mi admiración y reconocimiento al co-autor de esta ponencia Dr. Xorge A. Domínguez por la paciente revisión, sugestiones y arreglo técnico hechos al manuscrito. Que los asistentes a la plática juzguen del valor de esta secuencia de datos que en modo alguno pretenden ser exhaustivos. La riqueza bibliográfica en fitoquímica a nivel mundial es asombrosa y ello habla por sí solo de la importancia de este vasto campo del saber humano. Los autores agradecen al Dr. Eduardo Aguirre Pequeño el haber proporcionado el libro "Datos para la integración de la Materia Médica Nacional" (7) para su consulta; es una valiosa obra.

\subsection{LITERATURA CITADA}

1. Agureil, S. 1969. Identification of alkaloids intermediates by gas chromatographymass spectrometry. I. Potential mescaline precursors in Trichocereus spp. Lloydia 32: 40-45.

2. - 1969. Cactaceae alkaloids. I. Ibid. 32: 206-216.

3. - - J. G. Brunn, J. Lunstrom y U. Svensson. 1971. Cactaceae alkaloids. X. Alkaloids of Trichocereus species and some other cacti. Ibid. 34 (2) : 183-187.

4. Al-Shenbaz, I. A. 1973. The biosystematies of the genus Thelypodium (Cruciferae). Contr. Gray Herb. No. 204: 3-148.

5. Alston, R. E. y B. L. Turner. 1963. Biochemical Systematics. Prentice-Hall, Inc.

6. - T. J. Mabry y B. L. Turner. 1963. Perspectives in Chemotaxonomy. Science 142 (No. 3592): 545-552.

7. Altamirano, F., J. Ramírez, M. Toussaint, J. Terrés, E. Armendárriz, D. OrvaÑanos y S. Sosa. 1894. Datos para la integración de la Materia Médica Nacional. Primera parte. Inst. Médico Nacional. Secretaria de Fomento, pp. 1-140. México.

8. Barkley, F. A. y K. S. Boghdan. 1972. Herbarium specimens. The Gloxinian 22 (1): 29-32.

9. Beaman, J. H., R. C. Roluns y A. H. Smith. 1965. The herbarium in the modern University. A. Symposium. Taxon i4: 113-133.

10. Beschel, R. E. y J. H. Sopler. 1970. The automation and standardization of certain herbarium procedures. Can. J. Bot. 48 (3): 547-554.

11. Blomster, R. N., A. E. Schwarting y J. M. Berbitt. 1964. Alkaloids of Heimia salicifolía. J. A. preliminary report. Lloydia 27 (1): 15-14.

12. Bruhn, J. G. 1977 Alcaloides en las Cactáceas. Cact. Suc. Mex. 16 (3) : 51-58.

13. — 1973. Búsqueda etnobotánica de Cactáceas alucinógenas. Ibid. 18 (1): 8-13.

14. - y C. Brunn. 1973. Alkaloids and ethnobotany of Mexican peyote cacti and related species. Econ. Bot. 27 (2) : 241.251.

15. Caballero, Y. y F. Walls. 1970. Productos naturales del zoapatle (Montanoa tomentosa Cerv.). Bol. Inst. Quim. Univ. Nal. Aut. Mex. 22: 79-102.

16. Cava, M. P., S. K. Talapatra, K. Nomura, J. A. Weisbach, B. Douglas y E. C. 
Shoop. 1963. Haplocine and haplocidine: New aspidospermine-type alkaloids from Haplophyton cimicidium. Chem. Ind. 1963: 1242-1243.

17. - - P. Yates, M. Rosenberg, A. G. Szabo, B. Douglas, R. F. Raffauf, E. C. Shoop y J. A. Wisbach. 1963. Cimicine and cimicidine: lactonic akaloids of the aspidospermine skeletal type. Ibid.: 1875-1876.

18 - T. A. REED y J. L. Beal. 1965. An efficient separation of the common alkaloids of the berberine group; the isolation and characterization of columbamine. Lloydia 28 (1) : 73-83.

19. Correa, J. y J. Romo. 1966. The constituents of Cacalia decomposita A. Gray. Structures of maturine, maturinin, maturone and maturinone. Tetrahedron 22: 685-691.

20. Cromwell, B. T. 1933. Experiments on the origin and function of berberine in Berberis darwinii. Biochem. J. 27: 860-872.

21. Cruse, R. R. 1973. Desert plant chemurgy: a current review. Econ. Bot. 27 (2): 210-230.

22. Cuevas, L. A., F. García J. y A. Romo de Vivar. 1972. Estructura de la estenolobina. Rev. Lat. Quim. 3 (1): 22-27.

23. Chatterjee, R. y M. P. Guha. 1951. Studies on Mahonia. IV. Mahonia leschenaultii, M. manipurensis y M. sikkimensis. J. Am. Pharm. Assoc. 40: 229-232.

24. Dakshini, K. M. M. y F. Singh. 1970. Studies in the identification of Compositae taxa by paper chromatography. Phyton (Austria) 14 (1-2): 23-30 (una fig.).

25. De Lille, J. 1931. Contribución al conocimiento de la farmacología de la cereína. An. Inst. Biol. Mex. 2 (2) : 139-150.

26. ㄴ.. 1931. Nota acerca de la farmacología de la pachycereína. Ibid. 2 (3) : 219-221.

27. Djerassi, C., M. Gorman, A. L. Nussbaum y J. Reynoso. 1953. Alkaloid studies. II. Isolation of reserpine and narcotine from Rauwolfia heterophylla Roem. and Schult. J. Am. Chem. Soc. 75: 5446-5447.

28. - C. R. Smith, S. P. Marfey, R. N. McDonald, A. J. Lemin, S. K. Fidger y H. Estrada. 1954. Alkaloid studies. III. Isolation of piloceine and anhalonidine from four cactus species. Ibid. 76. 3215-3216.

29. ——— - S. K. Fidger, J. Herrán y J. Romo. 1954. Alkaloid studies. VI. Cuauchichicine, a new diterpenoid alkaloid. Ibid. 76: 5889.

30. —, P. Sengupta, J. Herrán y F. Walls. 1954. Terpenoids. V. The isolation of iresin, a new sesquiterpen-lactone. Ibid. 76: 2966-2968.

31. —- M. Gorman, A. L. Nussbaum y J. Reyonso. 1954. Alkaloid studies. IV. The isolation of reserpine, serpentine and ajmaline from Rauwolfia heterophylla Roem. and Schult. Ibid. 76: 4463-4465.

32. —- A. Bowers, S. Burstein, H. Estrada, J. Grossman, J. Herrán, A. J. Lemin, A. Manjarrez y S. C. Pakrashi. 1956. Terpenoids. XXII. Triterpenes from some Mexican and South American plants. Ibid. 78: 2312-2315.

33. - , S. Burstein, H. Estrada, A. J. Lemin, A. E. Lippman, A. Manjarrez y H. G. Monsimer. 1957. Terpenoids. XXVIII. The triterpene composition of the Genus $M y r$ tillocactus. Ibid. 79: 3525-3528.

34. Domínguez, E. y J. Romo. 1963. Mexicanin. I. A new sesquiterpene lactone related to tenulin. Tetrahedron 19: 1415-1421.

35. Domínguez, X. A., G. Leal D. y Ma. A. Vinales. 1958. Síntesis de N-isopropil y $\mathrm{N}$-isobutilamida de algunos ácidos y comparación de su acción insecticida con la afinina. Ciencia (Mex.) 17 (10-12) : 213-216.

36. - - P. Rojas, V. Collins y Ma. R. Morales. 1960. A phytochemical study of eight Mexican plants. Econ. Bot. 14. (2) : 157-159.

37. — 1962. Análisis fitoquímico. Ciencia (Mex.) 21 (3) : 125-135.

38. - R. Franco O., P. Rojas M., A. Elizondo, J. Valenzuela y E. Guajardo M. 1964. Determinación de la acción antibiótica de noventa y dos plantas mexicanas tóxicas al ganado o utilizadas con propósitos medicinales. Ibid. 23 (3): 99-103.

39. - - J. García D., A. Monroy C., L. G. Armendáriz, A. Alcalá, J. Quevedo y P. Rojas M. 1964. A chemical study of Bocconia latisepala Wats. Can. J. Chem. 43: 679-682. 
40. - - V. Barragín. 1965. Isolation and structure of Aeneadiol. J. Org. Chem. 30: 204.9-2050.

4.1. - P. Rojas M., Y. Almaguer y G. Hinojosa. 1965. Isolation of apocynine (acetovanillone) from two cacti. Chem. and Ind. 1965: 795.

42. - 1968. Industrialización racional de las plantas nativas de las zonas áridas. Depto. de Química. I.T.E.S.M. (Mimeografiado, 7 pp.).)

4.3. - y O. Pugliese C. 1967. A chemical study of Mammillaria runyoni. Planta Médica 15 (4) : 401-403.

44. - R. H. Ramírez, O. Lock Ugaz, J. García D. y R. Ketchum. 1968. Chemical study of the cactus Ariocarpus retusus. Ibid. 16 (2): 182-183.

45. - 1969. Chemical components of Leucophyllum texanum. Isolation of regiomontane. Ibid. 17 (4) : 366-368.

46. - - P. Rojas, M. Gutiérrez, N. Armenta y G. de Lara. 1969. Estudio químico preliminar de 31 cactáceas. Rev. Soc. Quim. Mex. 13 (I) : 8A-12A.

47. - M. Gutiérrez y N. Armenta. 1970. A Chemical survey of seventeen medicinal Mexican plants. Planta Medica 18 (I) : 51-54.

48. - 1970. Comparación de las substancias citotóxicas de origen vegetal con las plantas mexicanas usadas contra el cáncer y compuestos aislados de ellas. Rev. Asoc. Farmacéutica Mexicana 2 (6): 223-240.

49. $\longrightarrow$ y A. Sierra. 1970. Isolation of a new diterpene alcohol and parthenin from, Parthenium hysterophorus. Planta Medica 18 (3) : 275-277.

50. — S. Escarria y C. PÉrez E. 1970. Chemical studies of cacti. V. Constituents of the Coryphantha palmeri Britton-Rose and Echinocactus grandis Rose. Ibid. 18 (4): 315-317.

51. - Ma. E. Gómez, A. Gómez P., A. Nora Villarreal y C. Rombold. 1970. Physical data on the essential oils of five Compositae plants. Ibid. 19 (1) : 52-54.

52. $\longrightarrow$, B. E. Armas y F. Jáuregui. 1972. Estudio químico de Phoradendron coryae. Rev. Latin. Quim. 3 (I) : 28-30.

53. - y A. Merijanian. 1972. Constituents of the petroleum ether extract of Helietta parvifolia (A. Gray). Ibid.: 31.

54. - en colaboración con O. Ohno, H. Hirai, H. Yoshioka y T. J. Mabry. 1973. Compositae. Cyanopicrin: a sesquiterpene lactone from Centaurea americana. Phytochemistry 12: 221-222.

55. — 1973. Métodos para Investigación Fitoquímica. Limusa-Wiley.

56. — - S. Jiménez J. 1973. Aislamiento y estructuras del julslimterol, nor-julslimdiolona y la isocumambrina, metabolitos secundarios del Croptilon divaricatum (Compositae). Rev. Lat. Quim. 3 (4) : 177-182.

57. Douglas, B., J. L. Kirkpatrick, R. F. Raffauf, O. Ribeiro y J. A. Weisbach. 1964. Problems in Chemotaxonomy. II. The major alkaloids of the genus Heimia. Lloydia 27: 25-31.

(La parte I. de estos estudios apareció en J. Pharm. Sci. 1963, 52, 350).

58. Luperrex, A. 1969. L'importance des jardins botaniques pour l'enseignement de l'horticulture. Boissieria 14: 51-55.

59. Lstrada H., E. Estrada y L. Maya. 1965. Estudio de la Flourensia resinosa. Bol. Inst. Quim. Univ. Nal. Aut. Mex. 17: 68-75.

60. Flores, S. E. y J. Herkán. 1958. The structure of pendulin and penduletin: a new flavonol glucoside isolated from Brickellia pendula. 'T'etrahedron 2: 308-315.

61. $\frac{1}{1}$. 1960. Atanasin: a new flavone isolated from Brickellia squarrosa (Cav.) Rob. Chem. Ind. 1960: 291.

62. Fosberg, F. R. y M. H. Sachet. 1965. Manual for tropical herbaria. Reg. Veg. 39. Utrecht. (132 pp.).

63. García-Jiménez F. y M. C. Pérezamador. 1967. Corymbosin, a glucoside from Turbina corymbosa. Tetrahedron 23: 2557-2561.

64. Grbes, R. D. 1958. Chemical evolution in plants. J. Linn. Soc. London Zool. 44. (295); Bot. 56 (365) : 50-57 (citado por LuTin, ver No. 84). 


\section{FITOQUIMICA Y HERBARIOS}

65. Gómez-Pompa, A. 1962. Notas botánicas sobre algunas Dioscorea de importancia farmacéutica. Ciencia (Mex.) 21 (5-6) : 221-229.

66. - y L. I. Nevling, JR. 1973. The use of electronic data processing methods in the Flora of Veracruz program. Contr. Gray Herb. 203: 49-64.

67. González, Ma. P., P. Joseph.Nathan y J. Romo. 1971. Further advances in the chemistry and spectrometry of cacalone. Rev. Lat. Quim. 2:

68. Greathouse, G. A. y S. M. Watkins. 1938. Berberine as a factor in the resistance of Mahonia trifoliolata and Mahonia swaseyi to Phymatotrichum root rot. Am. J. Bot. 25: $743-748$.

69. Guerra, F. 1950. Bibliografía de la Materia Médica Mexicana. La Prensa Médica Mexicana. México.

70. - 1953. La investigación de las drogas mexicanas. Métodos y resultados. Mem. Congr. Cient. Mex. 10: 106-109.

71. Herz, W. y H. Watanabe 1959. Parthenin, a new guaianolide. J. Am. Chem. Soc. 81: 688.

72. — A. Romo de Vivar, J. Romo y N. Vishanathan. 1963. Constituents of Heleniım species. XIII. The structure of helenalin and mexicanain A. Ibid. 85: 19-26.

73. $\longrightarrow,-, \mathrm{y}-$. 1963. Idem. XV. Tetrahedron 19: 1359-1369.

74. Hinton, J. y J. Rzedowski. 1972. George B. Hinton, collector of plants in South western Mexico. J. Arn. Arb. 53 (2) : 14l-181.

75. Jensen, U. 1968. Serologische Beiträge zur Systematik der Ranunculaceae. Bot. Jahrb. 88 (2) : 204-268; Ibid. 88 (3) : 269-310.

76. Joseph-Nathan, P., J. Reyes y Ma. P. González. 1968. Contribution to the chemistry of perezone. Tetrahedron 24: 4007-4013.

77. - y Ma. P. González. 1969. Análisis conformacional de algunos sesquiterpenos por resonancia magnética nuclear doble. Rev. Soc. Quim. Mex. 13 (2): 57A-61A.

78. - Ma. C. Negrete y Ma. P. González. 1970. Studies in Cacalia species. Phytochemistry 9: 1623-1628.

79. - Ma. P. González, LeRoy F. Johnson y J. N. Shoolery. 1971. Natural abundance $13_{\mathrm{C}}$ NMR studies of perezone and derivates. Organic Magn. Reson. 3: 23-29.

80. - Ma. P. González y V. M. Rodríguez. 1972. Terpenoids of Perezia hebeclada. Phytochemistry 11: 1803-1808.

81. Kaufman, E. 1968. La industria esteroidal en México. "Editorial". Rev. Soc. Quim. Mex. $12(5): 211$ A.

82. Langman, I. K. 1964. A selected guide to the literature on the flowering plants of Mexico. Univ. Penn. Press.

83. Li, H. L. y J. J. Willaman. 1968. Distribution of alkaloids in Angiosperm Phylogeny. Econ. Bot. 22 (3) : 239-252.

84. Luten, D. B. 1964. On chemistry and taxonomy both biological and chemical. Lloydia 27 (2) : 135-137.

85. Mabry, T. J., A. Taylor y B. L. Turner. 1963. The betacyanins and their distribution. Phytochemistry 2: 61-64.

86. - J. KAgAN y H. Rösler. 1964. Nuclear magnetic resonance analysis of flavonoids. Univ. Texas Publ. No. 6418.

87. Maldonado, L. A., J. Herrán y J. Romo. 1966. La taliscanina, un componente de Aristolochia taliscana. Ciencia (Mex.) 24 (5-6): 237-240.

88. Manjarrez, A. y F. Medina. 1964. The analysis of the volatile oils of the leaves of Artemisia mexicana and A. klotzschiana. Can. J. Chem. 42: 2085-2088.

89. - y V. Mendoza. 1966. The volatile oils of Agastache mexicana (Benth.) Epling and Cunila lythrifolia Benth. Perf. and Essential Oil Rec. London.

90. Martínez, M. 1925. Plantas narcóticas de México. Bol. Direcc. Estud. Biol. Mex. 3: $21-26$.

91. —. 1969. Las plantas medicinales de México. Ediciones Botas. Mex. 656 pp.

92. Méndez, R. 1953. Farmacología de sustancias cardioactivas aisladas de plantas mexicanas. Mem. Congr. Cient. Mex. 10: 161-164.

93. Mirov, N. T. 1963. Chemistry and plant taxonomy. Lloydia 26: 117-124. 
94. Moss, G. P. 1971. The biogenesis of terpenoid essential oils. J. Soc. Cosmet. Chem. 22: 231-248.

95. Ortega, A., A. Romo de Vivar y J. Romo. 1968. Odoratin: a new pseudoguaianolide isolated from Hymenoxys odorata DC. Can. J. Chem. 46: 1539-1541.

96. - - G. Guerrero, A. Romo de Vivar, J. Romo y A. Palafox. 1971. La orizabina y la zexbrevina B, nuevos germacranólidos furánicos. Rev. Lat. Quim. 2:38-40.

97. - - G. Guerrrero y A. Romo de Vivar. 1973. Los componentes de Zexmenia brevifolia. III. Estructura de zexbrevina C. Rev. Lat. Quim. 4. (1): 1-7.

98. Padilla, J., J. Romo, E. Walls y P. Crabbé. 1967. Propiedades ópticas de unos derivados de la serie de la perezona y de los pipitzoles. Rev. Soc. Quim. Mex. 11 (1) : 7-11.

99. Payne, W. W., R. W. Scora y J. Kumanoto. 1972. The volatile oils of Ambrosia (Compositae: Ambrosieae). Brittonia 24 (2): 189-198.

100. - - Th. A. Geissman, A. J. Lucas y T. Saitoh. 1973. Chemosystematics and taxonomy of Ambrosia chamissonis. Bioch. System. 1: 21-33.

101. Pérezamador, M. C. y F. García J. 1966. Corymbol, new diterpenic alcohol. Tetrahedron 22: 1937-1942.

102. Petcru, Von P. 1968. Beiträge zur Phytochemie von Berberis dielsiana. Planta Medica 16 (4) : 421-425.

103. Plank, H. K. 1950. Insecticidal properties of some plants growing in Puerto Rico. U. S. Dept. Agr. Mayagüez, P. R. Bull. No. 49 (17 pp.).

104. Pomilio, A. B. 1973. Berberidaceae. Anthocyanins in fruits of Berberis buxifolia. Phy. tochemistry 12: 218-220.

105. Ponter, C. L. 1967. Taxonomy of Flowering Plants. W. H. Freeman Co.

106. Quijano, L., F. Malanco y T. Ríos. 1970. The structures of eupalin and eupatolin. Two new flavonol rhamnosides isolated from Eupatorium ligustrinum DC. Tetrahedron 26: $2851-2859$.

107. Raffauf, R. F. 1962. A simple field test for alkaloid containing plants, Econ. Bot. 16: 171-172.

108. Raffauf, R. F. 1970. Some notes on the distribution of alkaloids in the plant Kingdom. Ibid. 24 (1): 34-38.

109. Ramírez C., D. 1953. El herbario nacional, su historia y su importancia para el conocimiento de la flora mexicana. Mem. Congr. Cient. Mex. 6: 322-329.

110. Riba, R. 1969. El herbario nacional. Pasado, presente y futuro. Rev. Soc. Mex. Hist. Nat. 30: 25-37.

111. Rivera, I. 1943. Algunas plantas medicinales de Izúcar de Matamoros y pueblos anexos. An. Inst. Biol. Mex. 14 (1) : 37-67.

112. Ríos, T., A. Romo de Vivar y J. Rosio. 1967. Stevin, a new pseudoguaianolide isolated from Stevia rhombifolia H. B. K. Tetrahedron 23: 4265-4269.

113. Roca, J. 1931. Estudio químico preliminar del Pachycereus marginatus. An. Ins. Biol. Mex. 2 (2): 133-137.

114. Rodríguez Hahn, L., C. Sánchez y J. Rovo. 1965. Isolation and structure of jacquinic acid. Tetrahedron 21: 1735-1740.

115. _ A. Guzmán y J. Romo. 1968. The constituents of Cacalia decomposita A. Gray. 1V. Structure of decompositin. Ibid. 24: 477-483.

116. — y J. Rodríguez. 1973. Resinona, un nuevo triterpeno aislado de Fluorensia resinosa. Rev. Lat. Quim. 3 (4): 148-153.

117. Rojas Garcidueñas, M., X. A. Domínguez, J. Fernández y G. Alanís. 1972. New growth inhibitors from Parthenium hysterophous. Rev. Lat. Quim. 3 (2): 52-53.

118. Romo de Vivar, A., L. Rodríguez Hahn, J. Romo M. V. Lakshmikanthan, R. N. Mirrington, J. Kagan y W. Herz. 1966. Constituents of Helenium spp. XIX. Tetrahedron 22: 3279-3292.

119. - - y S. Figueroa. 1967. Contribución a la química de la ambrosina. Síntesis par. cial de partenina e isopartenina $(1,2)$. Rev. Soc. Mex. Quim. 11 (5): 146-148.

120. - y F. Obmos. 1968. Estudio cúmico de Achillea millefolium L. Ibid. 12 (5): 212 A. 213 A.

121. - y M. Aguilar. 3970. New pceudoguaianolide from Parthenium confertum Gray 


\section{FITOQUIMICA Y HERBARIOS}

(Compositae), Tetrahedron 26: 2775-2780 (en colaboración con H. Yoshioka, A. Hr. go, E. RodrígueZ, J. A. Mears y T. J. Mabry).

122. - L. A. Cuevas y C. Guerrero. 1971. Eupaglabrina, un nuevo terpeno aislado de Eupatorium glabratum. Rev. Lat. Quim. 2: 32-34.

123. - J. Guevara, C. Guerrero y A. Ortega. 1972. Los terpenoides de Mortonia greggii. Gray Estructura de la mortonina. A. Ibid. 3 (1): 1-4.

124. Romo, J., P. Josfph-Nathan y F. Díaz A. 1963. Aromatin and aromaticin, new ses. quiternene-lactones isolated from Helenium aromaticum (Hook.) Bailey. Chem. and Ind. 1963 , p. 1839.

125. — — - - 1964. The constituents of Helenium aromaticum (Hook.) Bailey. The structures of Aromatin and aromaticin. Tetrahedron 20: 79-85.

126. - y - 1964. The ronstituents of Cacalia decomposita A. Gray. Structures of ca. caliol and cacalone. Ibid. 20: 2331-2337.

127. - - - y G. Siade. 1966. The structure of cumanin, a constituent of Ambrosia cumanensis. Ibid. 22: 1499-1506.

128. - A. R. de Vivar v P. Josfrh-Nathan. 1967. The constituents of Zaluzania augusta. The structuers of zaluzanins A and B. Ibid. 23: 29-35.

129. —- - A. Vélfz y E. Urbina. 1968. Franserin and confertin: new nseudoguaianolides isnlated from Franseria and Ambrosia species. Can. I. Chem. 46: 1536-1538.

130. - T. Ríos v L. Quijano. 1968. Liøustrin, a guaianolide isolated from Eupatorium ligustrinum DC. Tetrahedron 24: 6087-6091.

131. ․ A. Romo dE. VIVAr y E. Díaz. 1968. The guaianolides of Ambrosia cumanensis H. B. K. The structures of cumarins A and B. Ibid. 24: 5625-5631.

132. — - - - - y A. VéLez, E. León y E. Urbina. 1970. The distribution of sesquiterpene lactones in several Ambrosia species. Rec. Adv. Phytochem. 3: 249-254.

133. - - - - y R. Treviño, P. Joseph-Nathan y E. Díaz. 1970. Constituents of Artemisia and Chrysanthemum species. The structure of chrysanthemins A and B. Phytochemistry 9: 1615-1621.

134. - y T. Rodríguez. Hahn. 1970. Canambrin, a new sesquiterpene dilactone from Ambrosia canescens. Ibid. 9: 1611-1614.

135. - - A. Romo de Vivar, A. Ortega y E. Díaz. 1970. Las guayulinas A y B, nuevos sesquiterpenos aislados del guayule. Rev. Lat. Quim. 1: 132-135.

136. - - H. Mundo y M. A. Cariño. 1972. Aislamiento y estructura de la nanamina, un componente de la Sweetia panamensis Benth. Rev. Lat. Quim. 3 (2): 46-49.

137. - y H. Truto. 1972. Estudin de Ta Artemisia mexirant. Armexina, un nuevo santauńlido cuva lactona nosee fusión cis. Ibid. 3 (3): 122-126.

138. - A. Romo df. Vivar y A. Ortega. 1973. Novel sesquiterpenes isolated in Compositac. Rec. Adv. Phytochem 6: 21-29.

139. Rosfythat.r. L. 1930. The chemical investigations of plants. G. Bell and Sons. Londres.

140. Sánchez Vifsca, F. v J. Rovo. 1963. Fstafiatina, a new sesquiterpene lactone isolatd from Artemisia mexicana. Tetrahedron 19: 1285-1291.

141. Sandovat. A. 1966. Cinco lustros de existencia. Bol. Inst. Química, Univ. Nal. Aut. Mex. 17: 83-121.

142. —- 1970. Mirada al origen del Boletín, 20 años después de publicación ininterrumnida. Ibid. 22: 3.9 .

143. Schultes, R. E. 1970 The botanical and chemical distribution of Hallucinogens. Ann. Rev. Plant Physiol. 21: 571-598.

144. - 1969. Hallucinogens of plant origin. Science 163: 245-254.

115. Sтм, S. K. 1964. Mediral plant elkaloide. An introduction for pharmacy students. Univ. Toronto Press. 181 pp.

146. - 1967. Medicinal plant glycosides. An introduction for pharmacy students, Ibid. $76 \mathrm{pp}$.

147. Sodi-Paldarts, F. 1961. Una sugestión para un nuevo agrupamiento de las plantas. Coct. Suc. Mex. 6 (1) : 12-15.

148. —. 1960. Alcaloides de las cactáceas. Ibid. 5 (2): 35-43. 
149. Solbric, O. T., T. F. Stuessy, B. L. Turner and coll. 1972. "Biochemistry and the Taxonomist". Prepared for the ASPT Symposium in Minneapolis, Minn. (References and lists of equipment for systematic studies with flavonoids, mustard oils, etc., including lists of references for each topic).

150. Sousa, M. 1969. Las colecciones botánicas de C. A. Purpus en Mexico. Univ. Calif. Publ. Bot. 51: i-ix y 1-36.

151. Tomita, M., Y. Inubushi y N. Mizoguchi. 1953. Alkaloids of berberidaceous plants. VI. Alkaloids of Mahonia japonica. J. Pharm. Soc. Japan 73: 776

152. Wallens, H., S. Serata y M. E. Walt. 1955. Steroidal sapogenins. XXXI. Gentrogenine and correllogenine, new sapogenins from Dioscorea spiculiflora. J. Am. Chem. Soc. 77 (19) : 5196-5197.

153. Walls, F., O. Collera y A. Sandoval L. 1958. Alkaloids from Stemmadenia spp. I. The alkaloids of $S$. donnellsmithii y $S$. galeottiana. Tetrahedron 2: 173-182.

154. Willaman, J. J. y B. G. Schubert. 1955. Alkaloid Hunting. Econ. Bot. 9 (2): 141-150.

155. - - 1961. Alkaloid-bearing plants and their contained alkaloids. U. S. Dept. Agric. Tech. Bull. No. 1234. Washington, D. C. 